\title{
A continuum model describing the reverse grain-size dependence of the strength of nanocrystalline metals
}

\author{
A. JÉRUSALEM $\dagger$, L. STAINIER $\$ \S$ and R. RADOVITZKY*† \\ $\dagger$ Department of Aeronautics and Astronautics, Massachusetts Institute \\ of Technology, 77 Massachusetts Ave, Cambridge, MA 02139, USA \\ †Département Aérospatiale \& Mécanique, LTAS-MCT, Université \\ de Liège, 1 Chemin des Chevreuils, B-4000 Liège, Belgium
}

\begin{abstract}
The breakdown of the Hall-Petch relation in the grain-size strength dependence of nanocrystalline metals has been rationalized by the activation of deformation mechanisms taking place at the grain boundary which compete with crystal plasticity and become dominant when grain sizes are sufficiently small. In this work, we develop a continuum description of the effective response of nanocrystalline metals. The model is based on a finite element formulation of the continuum three-dimensional problem describing the deformation of polycrystal grains explicitly and on the consideration of grain boundaries as surfaces of discontinuity with finite thickness embedded in the continuum. A phenomenological model formulated within the framework of variational constitutive updates is proposed to describe the operative grain-boundary deformation mechanisms of sliding and opening accommodation. The model parameters are fitted to atomistic results for copper at very high loading rate. Tensile test simulations using this model reproduce the inverse grain-size dependency of the macroscopic yield stress predicted by atomistic simulations. In particular, the model predicts that the grain-size dependency of the yield stress shows a linear relation to the inverse square root of the grain size, as in the traditional Hall-Petch law, but with a negative coefficient. The results are in good agreement with the atomistic simulations and also with quasi-static experimental tests. The ability to model the effective response of nanocrystals without the need of explicitly accounting for each individual atom opens the way for the analysis of nanocrystalline materials in sample sizes and under strain rates of technological significance.
\end{abstract}

\section{Introduction}

Nanocrystalline metals, i.e. metals with a polycrystalline structure and grain sizes in the nanometre range, have recently elicited significant interest because of their potential for achieving higher material strength, among other attractive mechanical

\footnotetext{
*Corresponding author. Email: rapa@mit.edu $\S$ Research associate at the Belgian Fonds National de la Recherche Scientifique.
} 
and electrical properties [1-6]. Efforts to characterize and understand the mechanical behaviour of these materials have unveiled some unique features of deformation that are not commonly observed in polycrystals.

Polycrystals with grain sizes in the micron range have long been known to exhibit a strong dependence of the yield stress on the grain size. This behaviour has been observed to agree with the Hall-Petch [7, 8] relation:

$$
\sigma_{y}=\sigma_{0}+k d^{-(1 / 2)}
$$

where $k$ is a positive multiplicative constant and $\sigma_{0}$ is the lattice friction stress. Based on this observation, microstructure refinement has been exploited as a means of producing materials with increased strength. There has been interest in elucidating the limits of the Hall-Petch relation and the implications on the ductility of the material. Starting with the pioneering experiments of Chokshi et al. [9], there has been experimental evidence that the yield stress decreases when the grain size is reduced beyond a given threshold [10-12]. In particular, it has been suggested that the dependence of the yield stress on grain size may follow an inverse square root relation, as in equation (1), but with a negative coefficient $k$. However, the experimental evidence is not conclusive and this aspect of the response of nanocrystals is still the subject of considerable debate [13]. As a result, there have been significant efforts to confirm and explain this inverse - sometimes also referred to as reverse - Hall-Petch effect in nanocrystals.

The widely accepted explanation of the direct Hall-Petch (HP) effect lies in the role played by pile-ups of lattice dislocations at the grain boundaries as a mechanism enhancing the resistance to plastic flow. As the grain size decreases, there is less room for dislocation activity inside the grains, whereas the relative volume fraction of grain boundary atoms increases. This suggests a qualitative change in the operative deformation mechanisms from dislocation-mediated plasticity to grain-boundary deformation mechanisms. Different grain-boundary deformation mechanisms explaining this qualitative change of behaviour have been proposed. Chokshi et al. suggested that Coble creep at room temperature is perhaps the mechanism explaining their experimental results [9]. But more recent molecular dynamics simulations of the yield response of nanocrystalline copper have shown a reverse Hall-Petch Effect in the absence of thermally activated processes [14].

Atomistic simulations [14-18] have shown that the main deformation mechanism taking place at grain boundaries consists of localized sliding accompanied by some accommodation mechanism that maintains the intergrain compatibility, e.g. at triple points. However, there is still dissent on the nature of this accommodation process. Recent large-scale atomistic simulations have been able to show the crossover from the direct to the inverse grain-size dependency of material strength [19].

There have also been efforts to describe deformation and failure mechanisms in nanocrystalline metals using continuum models. A summary of the literature in this area has been recently presented in the review article by Meyers et al. [13]. Continuum modelling approaches have included the development of mixed-phase constitutive models which consider the grain interior and boundary processes in a homogenized way [20-22], the explicit consideration of the grain boundaries as a continuum region with different properties [23-25] or by means of interface elements 
with a finite thickness in the finite element mesh [26, 27]. These studies have been limited to two-dimensional analyses focused on the ability of the continuum approach to describe grain size dependency [25-27], strain localization [24, 27] and failure $[25,26]$.

In this work, we extend this continuum approach to three dimensions and propose a continuum model describing the competing deformation mechanisms that are believed to determine the effective response of nanocrystalline (nc) materials. The main interest is in developing a continuum model describing the salient features of the macroscopic response of nc metals predicted by atomistic simulation. The model consists of a finite element formulation of the continuum three-dimensional problem describing the polycrystal grains explicitly and where grain boundaries are treated as discontinuity surfaces embedded in the continuum. Following what has been observed experimentally, grain boundaries are considered as having a small but finite thickness. The assumed grain boundary thickness, in effect, introduces a length scale in the model. Interface elements inspired on well-established descriptions of fracture and crack propagation [28] are formulated to account for the special kinematics of grain boundaries (i.e. to describe grain-boundary sliding and other accommodation mechanisms). The amount of grain-boundary sliding and accommodation is determined by a phenomenological model formulated within the framework of variational constitutive updates [29, 30], considering uncoupled plastic dissipative deformation mechanisms as the constitutive behaviour of grain boundaries. In essence, the model considers the grain boundary as a slip plane in a similar manner to crystal plasticity models but without a preferred slip direction. The opening mode is modelled with a similar plasticity formulation. Grain-boundary friction can be incorporated in the model in a straightforward manner [26]. However, in this work, friction was left unmodelled due to the lack of experimental or atomistic data available for calibration purposes. A similar situation was faced in the cited reference, where the friction coefficient was assumed to be zero. In our submission, all the data obtained from the literature corresponds to tensile loading conditions in which friction is expected to play a minor role, if any. Schuh et al. [31] address the issue of friction by comparing molecular statics simulations in tension and compression as well as in multiaxial loading conditions. However, the compressive response in this reference may be affected by the tetrakaidecahedral structures used in the simulations which has faces normal to the compressive loading axis. The compressive stress strain curves are then probably affected by the plastic response of the grains and not just by the increased sliding resistance of the grain boundary under compression due to friction. In a subsequent paper, we will report on our efforts to incorporate friction in the model for the purpose of describing the shock response of nc copper as recently investigated by Bringa et al. [32] using atomistic models. The model parameters are calibrated using experimental [33, 34] and atomistic [14] results for nanocrystalline copper from the literature. The resulting model is used to conduct numerical simulations of the tensile response of nanocrystalline copper for different grain sizes. The ability to use rather large computational meshes enables the investigation of grain-size dependency of the nanocrystal effective response while resolving the deformation field inside each grain as well as the grain interactions. To this end, tensile tests simulations of cubic-shape, $100 \mathrm{~nm}$ and $20 \mathrm{~nm}$ sized nanocrystalline copper specimens are conducted with grain sizes ranging 
from $33.33 \mathrm{~nm}$ to $3.33 \mathrm{~nm}$. The numerical results are compared to both experimental $[33,34]$ and atomistic results [14]. The model is found to capture the reverse Hall-Petch size dependency in good agreement with atomistic simulations.

The continuum modelling framework for polycrystals including the governing equations, the kinematic assumptions and the variational constitutive framework are presented in section 2. The numerical approximation is described in detail in section 3 . Section 4 is devoted to the presentation of numerical results, which include the constitutive parameter fit to atomistic data and the study of size-dependency of the yield stress of nanocrystalline copper under very high deformation rates and quasi-static conditions. A summary and conclusions of the paper are presented in section 5 .

\section{Continuum formulation}

\subsection{Governing equations}

We assume from the outset that the kinematics of the deformation mechanisms taking place at grain boundaries may be described as surfaces of discontinuity embedded in an otherwise continuous medium. Towards this end, we adopt the continuum framework described in [28], which we briefly summarize in this section. The undeformed polycrystal lies in the region of space occupied by the set $B_{0}=\bigcup_{g} B_{0}^{g} \subset \mathbb{R}^{3}$, where $B_{0}^{g}$ is the region occupied by grain $g$, and undergoes a motion $\varphi: B_{0} \times[0, t] \rightarrow \mathbb{R}^{3}$, where $[0, t]$ is the time interval considered. In the continuous regions of the polycrystal, i.e. the grain interiors, local information about the material deformation is conveyed by the deformation gradients $\mathbf{F}$, whereas the local stress state is described by the first Piola-Kirchhoff stress tensor $\mathbf{P}$ [35]. Figure 1 shows a representative intergrain boundary surface $\partial B_{0}^{g b}$ whose unit normal is $\mathbf{N}$. Considering a grain in isolation, the power expended by the boundary tractions $\mathbf{t}$, which includes both the external as well as the intergranular tractions, is given by the expression:

$$
\int_{\partial B_{0}^{g}} \mathbf{t} \cdot \dot{\boldsymbol{\varphi}} d S_{0}
$$

where $\partial B_{0}^{g}$ includes the externally exposed $\partial B_{0}^{\text {ext }}$ (if any) as well as the intergrain $\partial B_{0}^{g b}$ portions of the grain boundary, i.e. $\partial B_{0}^{g}=\partial B_{0}^{\text {ext }} \cup \partial B_{0}^{g b}$. The total power of the surface tractions on the polycrystal is:

$$
\sum_{g} \int_{\partial B_{0}^{g}} \mathbf{t} \cdot \dot{\boldsymbol{\varphi}} d S_{0}=\int_{\partial B_{0}} \mathbf{t} \cdot \dot{\boldsymbol{\varphi}} d S_{0}+\underbrace{\sum_{g b \pm} \int_{\partial B_{0}^{g b \pm}} \mathbf{t} \cdot \dot{\boldsymbol{\varphi}} d S_{0}}_{\dot{W}_{g b}}
$$

where $\partial B_{0}=\bigcup_{g} \partial B_{0}^{\text {ext }}$ is the external boundary of the aggregate polycrystal, $\dot{W}_{g b}$ represents the deformation power associated with the grain boundary and the \pm sign indicates that the surface integral is to be computed on both sides of the intergrain boundaries. 


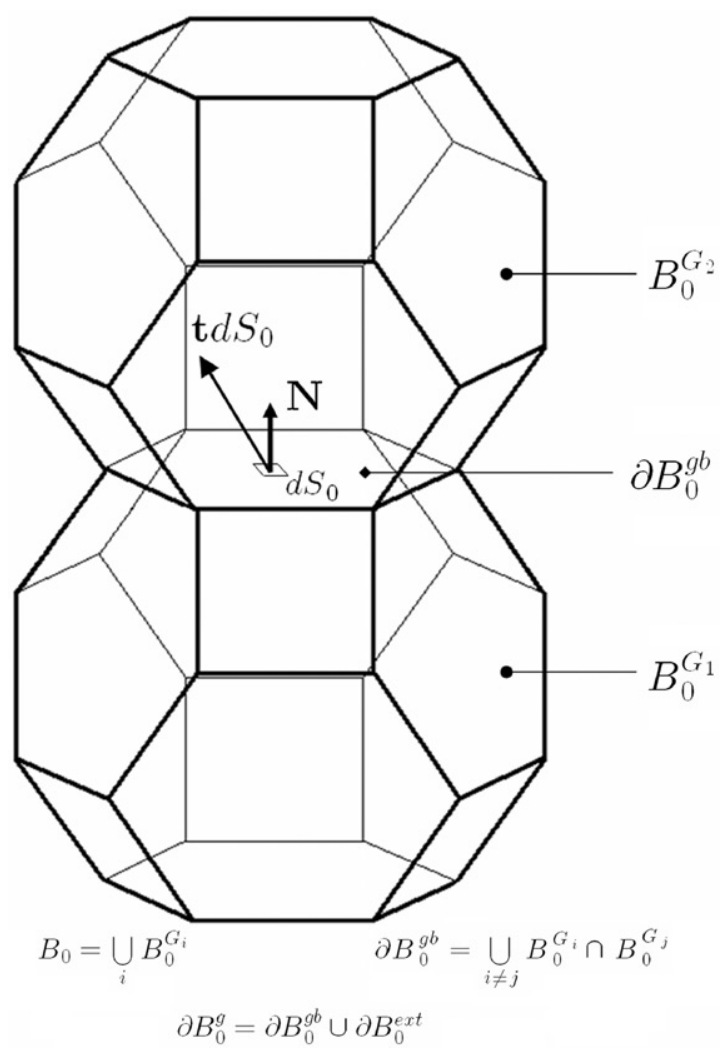

Figure 1. Schematic of geometric model of nanocrystals as a continuum with surfaces of discontinuity at grain boundaries.

Inspired by atomistic simulations showing that grain-boundary sliding and other accommodation mechanisms become operative in the deformation of nanocrystals [14-18], here and subsequently we allow for discontinuous displacements across the grain boundaries. Equation (3) then shows that the resulting finite displacement jumps $\delta=\llbracket \varphi \rrbracket=\varphi^{+}-\varphi^{-}$at the grain boundary constitute the appropriate deformation measure that is work-conjugate to the intergrain boundary tractions. In addition, balance of linear momentum requires $\mathbf{P} \cdot \mathbf{N}=\mathbf{t}$ on $\partial B_{0}^{g}$ and the tractions to be continuous across the intergrain boundaries, i.e. $\llbracket \mathbf{t} \rrbracket=\llbracket \mathbf{P} \cdot \mathbf{N} \rrbracket=\mathbf{0}$. The total power of the external loads including the body forces $\rho_{0} \mathbf{b}$ is therefore:

$$
\begin{aligned}
\dot{W} & =\int_{B_{0}} \rho_{0} \mathbf{b} \cdot \dot{\boldsymbol{\varphi}} d V_{0}+\int_{\partial B_{0}} \mathbf{t} \cdot \dot{\boldsymbol{\varphi}} d S_{0}+\sum_{g b} \int_{\partial B_{0}^{g b}} \mathbf{t} \cdot \llbracket \dot{\boldsymbol{\varphi}} \rrbracket d S_{0} \\
& =\int_{B_{0}} \boldsymbol{g}\left(\rho_{0} \mathbf{b}+\nabla_{0} \cdot \mathbf{P}\right) \cdot \dot{\boldsymbol{\varphi}} d V_{0}+\int_{B_{0}} \mathbf{P} \cdot \dot{\mathbf{F}} d V_{0}+\sum_{g b} \int_{\partial B_{0}^{g b}} \mathbf{t} \cdot \llbracket \dot{\boldsymbol{\varphi}} \rrbracket d S_{0} .
\end{aligned}
$$


The deformation power is the portion of the external power not consumed in increasing the kinetic energy $K=\sum_{g} \int_{B_{0}^{g}} 1 / 2 \rho_{0}|\dot{\varphi}|^{2} d V_{0}$ :

$$
P^{D}=\dot{W}-\dot{K}=\int_{B_{0}} \mathbf{P} \cdot \dot{\mathbf{F}} d V_{0}+\underbrace{\sum_{g b} \int_{\partial B_{0}^{g b}} \mathbf{t} \cdot \llbracket \dot{\varphi} \rrbracket d S_{0}}_{\dot{W}_{g b}}
$$

where linear momentum balance $\nabla_{0} \cdot \mathbf{P}-\rho_{0}(\ddot{\boldsymbol{\varphi}}-\mathbf{b})=0$ inside the grains $B_{0}^{g}$ has been imposed. It becomes clear from equation (5) that in this polycrystal modelling framework, the deformation power encompasses the contributions of the internal stresses inside each grain, as in conventional continuous solids, as well as those of the intergranular tractions which are work-conjugate to the displacement jumps describing the deformation mechanisms at the intergrain boundaries.

Taking as a starting point the work conjugacy relations suggested by the deformation power identity, equation (5), we develop a constitutive framework for the mechanical behaviour of nanocrystalline materials including grain-boundary deformation mechanisms.

Atomistic simulation studies have shown that dislocation-mediated plastic deformation inside the grains in nanocrystalline materials is ostensibly impeded $[17,19]$. Consequently and in order to reveal the independent role played by grain-boundary deformation mechanisms, we will assume that the grain interiors can only undergo a reversible, i.e. elastic, lattice distortion and rotation. The competition between intra-grain crystal plasticity and grain-boundary deformation has been discussed in $[26,27]$. As the distortion of the lattice is expected to be small, a linear anisotropic relation between the Lagrangian strain tensor $\mathbf{E}=(\mathbf{C}-\mathbf{I}) / 2$ and the second Piola-Kirchhoff $\mathbf{S}=\mathbf{F}^{-1} \mathbf{P}$ is assumed in which the first order elastic moduli are obtained directly from the crystal elastic constants. In these expressions, $\mathbf{C}=\mathbf{F}^{T} \mathbf{F}$ is the right Cauchy-Green deformation tensor and $\mathbf{I}$ is the unit tensor.

As suggested by equation (5), the displacement jumps across the grain boundary $\boldsymbol{\delta}$ are taken as the kinematic variable or deformation measure, whereas the grain-boundary traction vector $\mathbf{t}$ is taken as the corresponding work-conjugate stress measure. It should be noted that $\boldsymbol{\delta}$ vanishes for a rigid body motion, as should be the case for an appropriate deformation measure.

\subsection{Kinematic assumptions}

For the purpose of tracking geometric information of the deformed grain-boundary surface, we conventionally define the mean deformation mapping [28]:

$$
\tilde{\varphi}=\frac{1}{2}\left(\varphi^{+}+\varphi^{-}\right)
$$

from which the original deformation mapping on both sides of the grain boundary can be recovered as:

$$
\varphi^{ \pm}=\tilde{\varphi} \pm \frac{1}{2}\left(\varphi^{+}-\varphi^{-}\right)=\tilde{\varphi} \pm \frac{1}{2} \delta
$$


Thus, the deformed grain boundary is defined as $S \equiv \tilde{\varphi}\left(S_{0}\right)$. Given a parameterization $\tilde{\boldsymbol{\varphi}}=\tilde{\boldsymbol{\varphi}}\left(s_{\alpha}\right), \alpha=1,2$ of $S$, as subsequently provided by the finite element discretization of the grain boundaries, the deformed surface normal $\mathbf{n}$ may be obtained directly from the covariant basis vectors $\mathbf{a}_{\alpha}=\tilde{\boldsymbol{\varphi}}_{, \alpha}$ as:

$$
\mathbf{n}=\frac{\mathbf{a}_{1} \times \mathbf{a}_{2}}{\left\|\mathbf{a}_{1} \times \mathbf{a}_{2}\right\|} .
$$

The displacement jumps may then be decomposed into an opening separation vector and a grain-boundary sliding vector as follows:

$$
\begin{aligned}
& \boldsymbol{\delta}_{n}=(\boldsymbol{\delta} \cdot \mathbf{n}) \mathbf{n}=(\mathbf{n} \otimes \mathbf{n}) \cdot \boldsymbol{\delta} \\
& \boldsymbol{\delta}_{s}=\boldsymbol{\delta}-\boldsymbol{\delta}_{n}=(\mathbf{I}-\mathbf{n} \otimes \mathbf{n}) \cdot \boldsymbol{\delta} .
\end{aligned}
$$

In addition, the mean deformation of the grain-boundary surface may be measured by the material gradient of the mean deformation mapping $\nabla_{S_{0}} \tilde{\varphi}$. It is worth noting that the mean deformation of the grain-boundary surface $\nabla_{S_{0}} \tilde{\varphi}$ describes in-plane stretching and shearing deformation of the grain boundary in the absence of relative grain sliding or opening. In particular, the surface deformation gradient conveys information about the normal, equation (8), and the surface deformation as measured by the surface Cauchy-Green deformation tensor $\mathbf{C}_{S_{0}}=\left(\nabla_{S_{0}} \tilde{\varphi}\right)^{T} \nabla_{S_{0}} \tilde{\boldsymbol{\varphi}}$. This information would be required in models accounting for the influence of grain boundary misorientation or preferred sliding directions. However, due to the absence of supporting experimental evidence such additional complexities will be ignored.

These kinematic assumptions lead to a constant state of deformation across the thickness $h$ of the grain boundary which can be expressed in the local orthonormal reference frame $\left(\mathbf{n}_{1}, \mathbf{n}_{2}, \mathbf{n}_{3}\right)=\left(\left(\mathbf{a}_{1} /\left|\mathbf{a}_{1}\right|\right),\left(\mathbf{n} \times \mathbf{a}_{1} /\left|\mathbf{n} \times \mathbf{a}_{1}\right|\right), \mathbf{n}\right)$ as:

$$
\boldsymbol{\epsilon}=\underbrace{\frac{\boldsymbol{\delta}_{n} \cdot \mathbf{n}_{3}}{h} \mathbf{n}_{3} \otimes \mathbf{n}_{3}}_{\boldsymbol{\epsilon}_{n}}+\underbrace{\frac{\boldsymbol{\delta}_{s} \cdot \mathbf{n}_{1}}{h} \frac{1}{2}\left(\mathbf{n}_{1} \otimes \mathbf{n}_{3}+\mathbf{n}_{3} \otimes \mathbf{n}_{1}\right)+\frac{\boldsymbol{\delta}_{s} \cdot \mathbf{n}_{2}}{h} \frac{1}{2}\left(\mathbf{n}_{2} \otimes \mathbf{n}_{3}+\mathbf{n}_{3} \otimes \mathbf{n}_{2}\right)}_{\boldsymbol{\epsilon}_{s}} .
$$

It should be noted that $h$ introduces a characteristic length scale in the model. The above expression also shows that the strain tensor additively decomposes in a sliding part $\boldsymbol{\epsilon}_{s}$ and a normal opening part $\boldsymbol{\epsilon}_{n}$.

\subsection{Variational constitutive framework}

For the purpose of formulating constitutive models of grain-boundary response and their corresponding algorithmic updates, we adopt the variational framework proposed in [29, 30]. It was shown in [29] that an incremental, pseudo-elastic strain energy density can be derived for a rather general class of elastic and inelastic materials, leading to algorithmic updates possessing a potential structure. An important advantage of this approach is that it naturally furnishes solutions of the incremental boundary value problem as the result of a variational statement. This approach was extended in [30], where, in addition, variational updates are presented 
for specific material models, including finite-deformation isotropic plasticity and single-crystal plasticity. More recently, the same framework has been used for developing models of non-cohesive granular media [36], porous plasticity [37], nonlinear viscoelasticity [38] and thermomechanics [39].

An internal variable formalism [40, 41] is adopted to describe inelastic processes and a decoupled response in the opening and sliding components is postulated, i.e. that opening deformations do not cause sliding tractions, and vice versa. The free energy density may thus be additively decomposed into a sliding part $A_{s}$ and an opening part $A_{n}$ :

$$
A\left(\boldsymbol{\epsilon}_{s}, \boldsymbol{\epsilon}_{n}, \mathbf{q}\right)=A_{s}\left(\boldsymbol{\epsilon}_{s}, \mathbf{q}\right)+A_{n}\left(\boldsymbol{\epsilon}_{n}, \mathbf{q}\right)
$$

where $\mathbf{q} \in \mathbb{R}^{n}$ denotes a suitable set of internal variables. In the following, for simplicity of notation, we will drop the subscripts $s$ and $n$, and treat both parts (sliding and opening) in an identical way.

We will consider that the material in the grain boundary undergoes an elasto-visco-plastic behaviour. We will further assume that the strain additively decomposes in an elastic and a plastic part:

$$
\boldsymbol{\epsilon}=\boldsymbol{\epsilon}^{e}+\boldsymbol{\epsilon}^{p}
$$

which, given the linearity of relation (11), amounts to an additive decomposition of the displacement jump $\boldsymbol{\delta}=\boldsymbol{\delta}^{e}+\boldsymbol{\delta}^{p}$. We also define the equivalent plastic strain $\bar{\epsilon}^{p}$, linked to the tensorial plastic strain through the flow rule:

$$
\dot{\boldsymbol{\epsilon}}^{p}=\dot{\bar{\epsilon}}^{p} \mathbf{M}
$$

where $\mathbf{M}$ is a symmetric tensor satisfying the following set of conditions $\left(J_{2}\right.$ plasticity):

$$
\left\{\begin{array}{c}
\operatorname{tr}[\mathbf{M}]=0 \\
\mathbf{M} \cdot \mathbf{M}=\frac{3}{2}
\end{array}\right.
$$

and otherwise undefined at this point. The set of internal variables is then $\mathbf{q}=\left\{\boldsymbol{\epsilon}^{p}, \bar{\epsilon}^{p}\right\}$ and will be determined by the variational principle described below. It will be assumed that the elastic response of the grain boundary is not affected by irreversible processes, as it is usually done for the elastic response of the crystal, and, thus, we can write

$$
A\left(\boldsymbol{\epsilon}, \boldsymbol{\epsilon}^{p}, \bar{\epsilon}^{p}\right)=W^{e}\left(\boldsymbol{\epsilon}-\epsilon^{p}\right)+W^{p}\left(\epsilon^{p}, \bar{\epsilon}^{p}\right) .
$$

Taking into account the flow rule, the thermodynamic forces associated to $\epsilon$ and $\bar{\epsilon}^{p}$ are respectively given by:

$$
\begin{aligned}
\boldsymbol{\sigma} & =\frac{\partial A}{\partial \boldsymbol{\epsilon}}=\frac{\partial W^{e}}{\partial \boldsymbol{\epsilon}^{e}} \\
Y & =-\frac{\partial A}{\partial \bar{\epsilon}^{p}}=\left(\boldsymbol{\sigma}-\boldsymbol{\sigma}^{c}\right) \cdot \mathbf{M}-\sigma_{y}
\end{aligned}
$$


where $\boldsymbol{\sigma}^{c}=\partial_{\epsilon^{p}} W^{p}\left(\epsilon^{p}, \bar{\epsilon}^{p}\right)$ is the backstress tensor and $\sigma_{y}=\partial_{\bar{\epsilon}^{p}} W^{p}\left(\epsilon^{p}, \bar{\epsilon}^{p}\right)$ is the yield stress corresponding to stored hardening mechanisms (by contrast to dissipative mechanisms). Finally, kinetic relations between $Y$ and $\dot{\bar{\epsilon}}^{p}$ are given by a convex dissipation pseudo-potential $\psi^{*}$ (convexity ensures positive dissipation):

$$
Y=\frac{\partial \psi^{*}}{\partial \dot{\bar{\epsilon}}^{p}}\left(\dot{\bar{\epsilon}}^{p} ; \bar{\epsilon}^{p}\right)
$$

If we define the following functional [30]:

$$
D=\dot{A}+\psi^{*}=\boldsymbol{\sigma} \cdot \dot{\boldsymbol{\epsilon}}-Y \dot{\bar{\epsilon}}^{p}+\psi^{*}\left(\dot{\bar{\epsilon}}^{p} ; \bar{\epsilon}^{p}\right)
$$

the constitutive relations are equivalent to the variational principle

$$
D^{\mathrm{eff}}(\dot{\boldsymbol{\epsilon}} ; \boldsymbol{\epsilon}, \mathbf{q})=\inf _{\dot{\bar{\epsilon}}^{p}, \mathbf{M}} D\left(\dot{\boldsymbol{\epsilon}}, \dot{\bar{\epsilon}}^{p}, \mathbf{M} ; \boldsymbol{\epsilon}, \mathbf{q}\right)
$$

Minimization of $D$ with respect to $\mathbf{M}$ corresponds to the principle of maximum plastic dissipation, and yields, using Lagrange multipliers to enforce conditions (15):

$$
\mathbf{M}=\sqrt{\frac{3}{2}} \frac{\mathbf{s}-\mathbf{s}^{c}}{\left\|\mathbf{s}-\mathbf{s}^{c}\right\|} \quad \text { where } \mathbf{s}=\operatorname{dev}[\boldsymbol{\sigma}] \text { and } \mathbf{s}^{\mathrm{c}}=\operatorname{dev}\left[\boldsymbol{\sigma}^{\mathrm{c}}\right]
$$

while the minimization with respect to $\dot{\bar{\epsilon}}^{p}$ yields the kinetic relations (19). An additional advantage of the variational formulation is that $D^{\mathrm{eff}}$ provides a rate-potential for the stress:

$$
\boldsymbol{\sigma}=\frac{\partial D^{\text {eff }}}{\partial \dot{\boldsymbol{\epsilon}}}(\dot{\boldsymbol{\epsilon}} ; \boldsymbol{\epsilon}, \mathbf{q})
$$

An incremental solution procedure that retains the variational properties is adopted to integrate the constitutive update following [30].

We consider here that grain-boundary sliding occurs exclusively by dissipative hardening micromechanisms. This implies that

$$
W^{p}\left(\epsilon^{p}, \bar{\epsilon}_{p}\right) \equiv 0
$$

In the specific model used in calculations, the following dissipation pseudo-potential is adopted

$$
\left\{\begin{array}{l}
\Psi^{*}\left(\dot{\bar{\epsilon}}_{p}, \bar{\epsilon}_{p}\right)=\tilde{\sigma}_{0}\left(\bar{\epsilon}_{p}\right) \dot{\epsilon}_{0} \frac{1}{m+1}\left(1+\frac{\dot{\bar{\epsilon}}_{p}}{\dot{\epsilon}_{0}}\right)^{m+1} \\
\tilde{\sigma}_{0}\left(\bar{\epsilon}_{p}\right)=\sigma_{0}\left(1+\frac{\bar{\epsilon}_{p}}{\epsilon_{0}}\right)^{n}
\end{array}\right.
$$

where $\epsilon_{0}, \dot{\epsilon}_{0}, \sigma_{0}, m$ and $n$ are model parameters. This expression leads to a power-law rate dependency and isotropic hardening model. 
By combining equations (18), (22), (24) and (25), we finally obtain:

$$
Y=\sqrt{\frac{3}{2}\left(s-s^{c}\right) \cdot\left(s-s^{c}\right)}=\tilde{\sigma}_{0}\left(\bar{\epsilon}_{p}\right)\left(1+\frac{\dot{\bar{\epsilon}}_{p}}{\dot{\epsilon}_{0}}\right)^{m} .
$$

With this constitutive framework in hand, the deformation power at the grain boundary (5) can be reformulated as

$$
\dot{W}_{g b}^{\mathrm{eff}}=\sum_{g b} \int_{\partial B_{0}^{g b}} D_{g b}^{\mathrm{eff}}(\dot{\boldsymbol{\delta}} ; \boldsymbol{\delta}, \mathbf{q}) d S_{0}
$$

in terms of the effective rate potential $D_{g b}^{\text {eff }}$ obtained by integrating (21) over the interface thickness using (11):

$$
D_{g b}^{\mathrm{eff}}(\dot{\boldsymbol{\delta}} ; \boldsymbol{\delta}, \mathbf{q})=\int_{-h / 2}^{h / 2} D^{\mathrm{eff}}(\dot{\boldsymbol{\epsilon}} ; \boldsymbol{\epsilon}, \mathbf{q}) d \zeta=h D^{\mathrm{eff}}(\dot{\boldsymbol{\epsilon}} ; \boldsymbol{\epsilon}, \mathbf{q}) .
$$

Using equation (11), equation (27) can then be simplified to:

$$
\dot{W}_{g b}^{\mathrm{eff}}=\sum_{g b} \int_{\partial B_{0}^{g b}} \frac{\partial D_{g b}^{\mathrm{eff}}(\dot{\boldsymbol{\delta}} ; \boldsymbol{\delta}, \mathbf{q})}{\partial \dot{\boldsymbol{\delta}}} \dot{\boldsymbol{\delta}} d S_{0}=\sum_{g b} \int_{\partial B_{0}^{g b}} h \frac{\partial D^{\mathrm{eff}}(\dot{\boldsymbol{\epsilon}} ; \boldsymbol{\epsilon}, \mathbf{q})}{\partial \dot{\boldsymbol{\delta}}} \llbracket \dot{\boldsymbol{\varphi}} \rrbracket d S_{0}
$$

Comparing this equation with (5) and using (23), the tractions are then given by:

$$
\mathbf{t}=h \frac{\partial D_{g b}^{\text {eff }}}{\partial \dot{\boldsymbol{\delta}}}(\dot{\boldsymbol{\epsilon}} ; \boldsymbol{\epsilon}, \mathbf{q})=h \boldsymbol{\sigma} \cdot \frac{\partial \boldsymbol{\epsilon}}{\partial \boldsymbol{\delta}}
$$

which can be simplified, using (11), to

$$
t=\left(\begin{array}{l}
\sigma_{13} \\
\sigma_{23} \\
\sigma_{33}
\end{array}\right)
$$

where the vector components are expressed in the local reference frame.

\section{Finite element discretization}

The continuum problem with embedded discontinuity surfaces representing the grain boundaries described above is approximated numerically using a conventional finite element discretization for the grain interiors and a discretization of the grainboundary deformation power based on grain-boundary interface elements. The geometric description of the interface elements follows [28]. In calculations, we use 10 -node tetrahedral elements for the continuum which naturally suggests grainboundary elements as schematically shown in figure 2 . The interface element consists of two six-node triangular surface elements lying on the positive $S^{+}$and negative $S^{-}$ sides of the grain boundary and offset along the normal by a distance equal to the grain boundary thickness $h$ in the reference configuration. Therefore, the total 


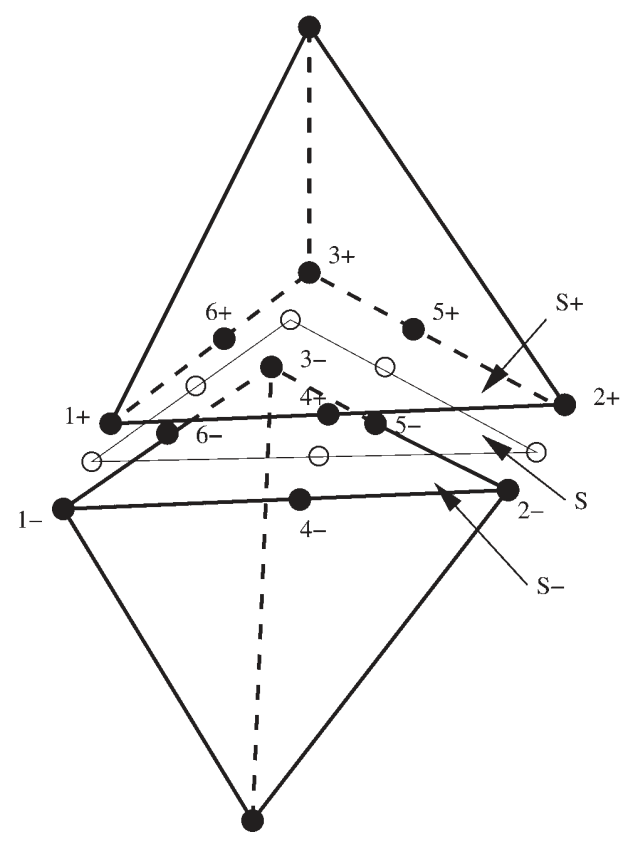

Figure 2. Schematic of grain-boundary element. Two tetrahedra belonging to two adjacent crystals separated by an interface element at the grain boundary: $S^{+}$and $S^{-}$are respectively the facets corresponding to the tetrahedra on the positive and negative side as defined by the positive surface normal $\mathbf{N}$ and $S$ is the midsurface.

number of nodes of the interface element is 12 . The nodes are numbered from 1 to 6 and identified with $+(-)$ if they lie on $S^{+}\left(S^{-}\right)$. The mean deformation mapping of the grain boundary $\tilde{\varphi}(6)$ is discretized using the standard shape functions for a sixnode triangular element $N_{a}\left(\xi_{1}, \xi_{2}\right), a=1, \ldots, 6$, and the mean nodal coordinates $\tilde{\mathbf{x}}_{a}=1 / 2\left(\mathbf{x}_{a}^{+}+\mathbf{x}_{a}^{-}\right)$, where $\mathbf{x}_{a}^{ \pm}$are the deformed nodal coordinates, as follows:

$$
\tilde{\boldsymbol{\varphi}}_{h}(\xi)=\sum_{a=1}^{6} N_{a}(\xi) \tilde{\mathbf{x}}_{a} .
$$

The isoparametric finite element interpolation of the middle surface of the interface element provides a parametric description of the element geometry in the deformed configuration from which all the geometric and kinematic quantities in section 2.2 can be conveniently obtained, including the deformed unit surface normal (8) which is computed using the covariant basis vectors:

$$
\mathbf{a}_{\alpha}(\xi)=\tilde{\boldsymbol{\varphi}}_{h, \alpha}(\xi)=\sum_{a=1}^{6} N_{a, \alpha}(\xi) \tilde{\mathbf{x}}_{a}
$$

and the interpolation of the displacement jumps:

$$
\boldsymbol{\delta}(\xi)=\llbracket \boldsymbol{\varphi}_{h} \rrbracket(\xi)=\sum_{a=1}^{6} N_{a}(\xi) \llbracket \mathbf{x}_{a} \rrbracket, \quad \llbracket \mathbf{x}_{a} \rrbracket=\mathbf{x}_{a}^{+}-\mathbf{x}_{a}^{-} .
$$


An application of the discretized values of the kinematic variables to the effective grain-boundary deformation power (27) leads to the expression of the internal grain-boundary forces contributed by each grain-boundary element:

$$
f_{I a}^{ \pm}=\mp \int_{S_{0}} t_{I} N_{a} d S_{0}
$$

with $\mathbf{t}$ given by (31), where $S_{0}$ is the reference configuration of the grain-boundary element and $N_{a}$, the conventional shape functions corresponding to quadratic isoparametric triangular elements as shown in figure 2. In this expression, thus, the node identifier $a$ ranges from 1 to 6 and the $+(-)$ sign applies to the nodes on the positive (negative) side of the grain-boundary element as informed by the positive surface normal resulting from the finite element parametrization. The stiffness matrix

$$
K_{I a K b}^{ \pm \pm}=\frac{\partial f_{I a}^{ \pm}}{\partial x_{K b}^{ \pm}}=\mp \int_{S_{0}} \frac{\partial t_{I}}{\partial x_{K b}^{ \pm}} N_{a} d S_{0}
$$

is required in static calculations and can be obtained after a straightforward derivation resulting in

$$
\begin{aligned}
K_{\text {IaK } b}^{ \pm \pm}= & \mp \pm \int_{S_{0}} \frac{n_{i I}}{2 h} \frac{\partial \sigma_{i 3}}{\partial \epsilon_{p l}}\left(\delta_{p 3} n_{l K}+\delta_{l 3} n_{p K}\right) N_{b} N_{a} d S_{0} \\
& \mp \int_{S_{0}} \frac{n_{i I}}{4 h} \frac{\partial \sigma_{i 3}}{\partial \epsilon_{p l}} \delta_{Q}\left(\delta_{p 3} \frac{\partial n_{l Q}}{\partial \bar{x}_{K b}}+\delta_{l 3} \frac{\partial n_{p Q}}{\partial \bar{x}_{K b}}\right) N_{a} d S_{0} \\
& \mp \int_{S_{0}} \frac{\sigma_{i 3}}{2} \frac{\partial n_{i I}}{\partial \bar{x}_{K b}} N_{a} d S_{0}
\end{aligned}
$$

where

$$
\frac{\partial n_{l Q}}{\partial \bar{x}_{K b}}=\lambda_{Q K b} \delta_{l 3}+\mu_{Q K b} \delta_{l 1}+v_{Q K b} \delta_{l 2}
$$

and

$$
\left\{\begin{array}{l}
\lambda_{I K b}=\frac{e_{K R S}}{\left|a_{1} \times a_{2}\right|}\left(a_{2 R} N_{b, 1}-a_{1 R} N_{b, 2}\right)\left(\delta_{I S}-n_{3 I} n_{3 S}\right) \\
\mu_{I K b}=\frac{N_{b, 1}}{\left|a_{1}\right|}\left(\delta_{I K}-\frac{a_{1 I} a_{1 K}}{\left|a_{1}\right|^{2}}\right) \\
v_{I K b}=e_{I J L}\left(\lambda_{J K b} n_{1 L}-\mu_{J K b} n_{3 L}\right)
\end{array}\right.
$$

In these expressions, capital indices correspond to the global reference frame and $\delta_{i j}$ and $e_{i j k}$ are respectively the Kronecker and the permutation symbols.

\section{Numerical investigations}

The computational framework for modelling the response of nanocrystalline metals presented in the foregoing is employed for investigating the tensile behaviour of 
nanocrystalline copper. Attention has been focused on nanocrystalline copper in the light of the availability of experimental and atomistic simulation data of the grain size dependence of strength in nanocrystals [10, 14, 17, 19, 21, 42, 43].

Idealized grain morphologies resulting from the three-dimensional packing of tetrakaidecahedra are adopted as the building blocks of the nanocrystalline samples for simplicity. This results in nanostructures consisting of equiaxed grains. Each grain is discretized using 192 second-order tetrahedral finite elements in a manner that leaves the overall polycrystal mesh conforming at the grain boundaries. Interface elements are added at the boundaries between grains, taking advantage of the conformity of the existing mesh. The idealized grain configurations are shown in figure 3 with their corresponding designations. The notation ' $1 \times 1 \times 1$ ' designates the microstructure with the fewest number of grains. This structure is a unit cell consisting of a full tetrakaidecahedron padded with eight grain eighths completing the regular cubic geometry. Similarly, the notation ' $1 \times n \times n$ ' designates a microstructure consisting of $n^{2}$ unit cells. This results in $n^{2}+2 \times(n+1)^{2}$ total number of grains including partial grains on the boundary.

Tensile test simulations are conducted by isostatically supporting the bottom face of the specimen and by imposing displacement boundary conditions on the top face. As mentioned in section 2, plastic deformation inside the grains is neglected. Furthermore, it is found that the elastic anisotropy of the grains does not significantly affect the macroscopic yield behaviour of the nanocrystalline sample, thus the grain interiors are assumed to follow an isotropic large deformation neo-hookean elastic law with Young's modulus $E=108 \times 10^{9} \mathrm{~Pa}$ and Poisson's ratio $v=0.3$. The properties of the grain-boundary model are calibrated against experimental and atomistic results as described below. One of the main issues that needs to be

(a)

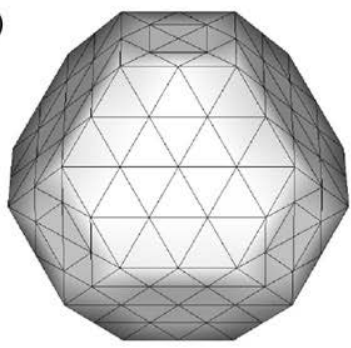

(b)

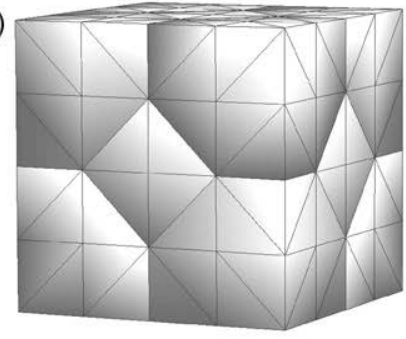

(c)

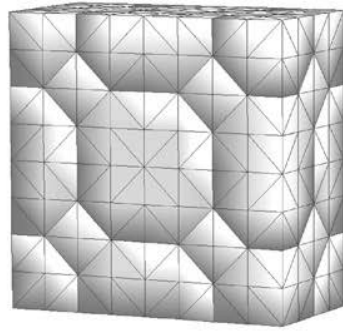

(d)

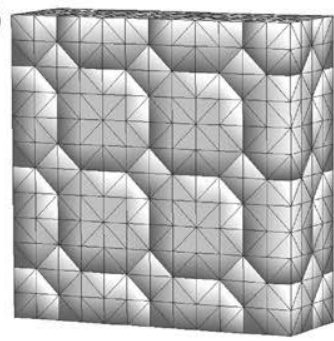

(e)

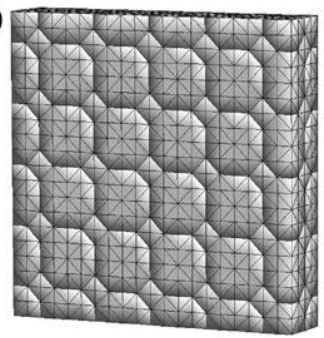

Figure 3. Idealized polycrystal grain configurations used in calculations. Designation and number of grains. 
confronted towards this end is the significant discrepancies in yield stress values between experiments and atomistic models [10, 14, 17, 19, 21, 42, 43]. These discrepancies have been attributed to the idealized polycrystalline configurations of the simulated samples including the lack of porosity and other defects, as well as to the unrealistic physics involved, which either considers extremely high strain rates or outright ignores thermally activated processes. It should be noted that molecular dynamics simulations under extreme rates $\left(5 \times 10^{8} \mathrm{~s}^{-1}\right)$ and molecular statics simulations are in relative good agreement in general (cf. for example [17] and [42]).

In this work, we attempt to fit representative atomistic and experimental results with a single set of constitutive parameters. It should be carefully noted that the physical phenomena built into the model such as rate-dependency does not necessarily provide a complete description of the actual physics involved.

The stress-strain curve corresponding to atomistic simulation of nanocrystalline copper with $6.56 \mathrm{~nm}$ mean grain size from [17] is used as a reference for calibration, as shown in figure 4. Tensile test simulations on cubic-shape samples of $20 \mathrm{~nm}$ per side with 91 grains and grain size $d=6.67 \mathrm{~nm}(3 \times 3 \times 3)$ were conducted at the same strain rate of $5 \times 10^{8} \mathrm{~s}^{-1}$ used in $[17,19]$. The elastic properties for bulk polycrystalline copper were adopted for the grain-boundary model and the yield, hardening and rate dependency parameters were adjusted until good agreement with the atomistic stress-strain curve was found. The comparison between the two and the resulting set of grain-boundary model parameters are shown in figure 4 and table 1 , respectively.

The same set of model parameters was used for simulating the experiments in $[33,34]$. The experimental stress-strain curve corresponding to grain size $d=26 \mathrm{~nm}$ [33] was used as a reference for comparison. The simulation was conducted using 66 grains of size $d=25 \mathrm{~nm}(1 \times 4 \times 4$ designation $)$ and under quasi-static conditions.

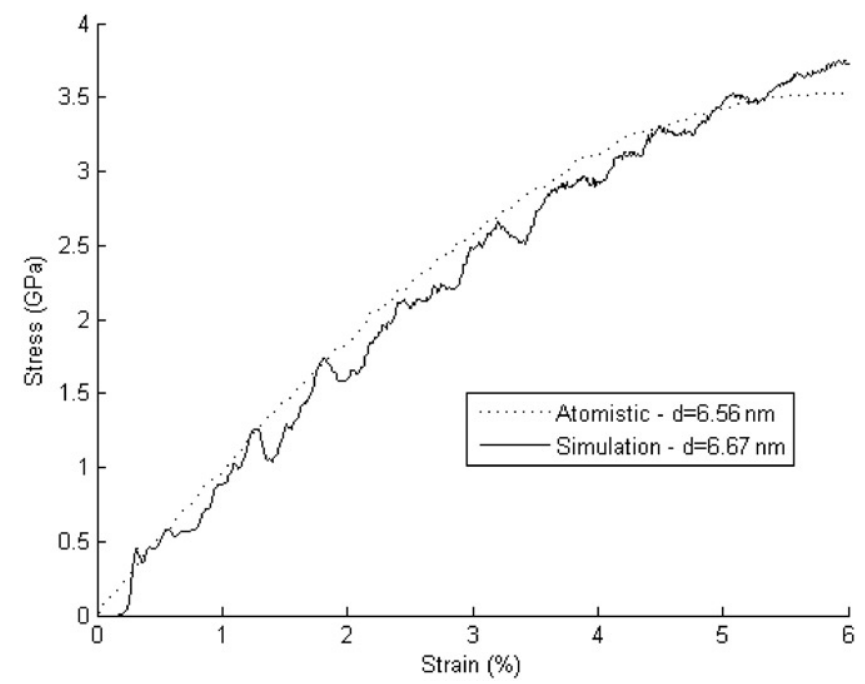

Figure 4. Calibration of grain-boundary model parameters against atomistic results from [17] for grain size $d \sim 6.6 \mathrm{~nm}$ and strain rate $5 \times 10^{8} \mathrm{~s}^{-1}$. 
Table 1. Calibrated grain-boundary model parameters for nanocrystalline copper.

\begin{tabular}{lc}
\hline Young's modulus $(\mathrm{Pa})$ & $108.0 \times 10^{9}$ \\
Poisson's ratio & $0.33 / 0$ (sliding/opening) \\
$\sigma_{0}(\mathrm{~Pa})$ & $200.0 \times 10^{6}$ \\
$\epsilon_{0}$ & $10^{-4}$ \\
$\dot{\epsilon}_{0}\left(\mathrm{~s}^{-1}\right)$ & 1.0 \\
$1 / n$ & 10 \\
$1 / m$ & 10 \\
$h(\mathrm{~nm})$ & 1 \\
\hline
\end{tabular}

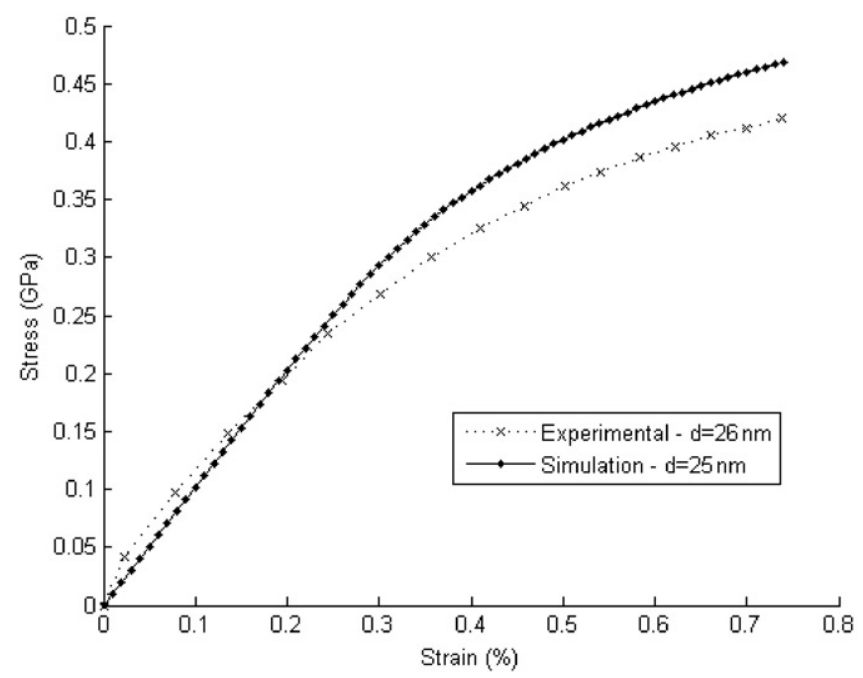

Figure 5. Comparison between tensile-test simulation for grain size $d=25 \mathrm{~nm}$ with experiments of Sanders et al. [33] using same set of model parameters calibrated to atomistic simulation results.

Figure 5 shows the comparison between the stress-strain curve obtained numerically and the experiment. Considering that a single set of model parameters is used, the agreement with the experiment is found to be reasonable. This suggests, in turn, that the discrepancy between experiments and atomistic simulations may be partially due to rate-dependency effects.

The calibrated model was then used to investigate the dependence of the stressstrain response on the grain size. In order to compare with the size-dependence results obtained from atomistic models in [14], a set of tensile test simulations was conducted keeping the sample size fixed at $20 \mathrm{~nm}$ and systematically increasing the number of grains from $22(1 \times 2 \times 2$ designation $)$ to $97(1 \times 5 \times 5$ designation $)$. This corresponds to grain sizes in the range $10 \mathrm{~nm}-4 \mathrm{~nm}$. In all the simulations, the grain-boundary thickness was kept at $h=1 \mathrm{~nm}$.

The resulting stress-strain curves are shown in figure 6. Owing to the extremely high strain rate $\left(5 \times 10^{8} \mathrm{~s}^{-1}\right)$ used in these simulations, significant dynamic 


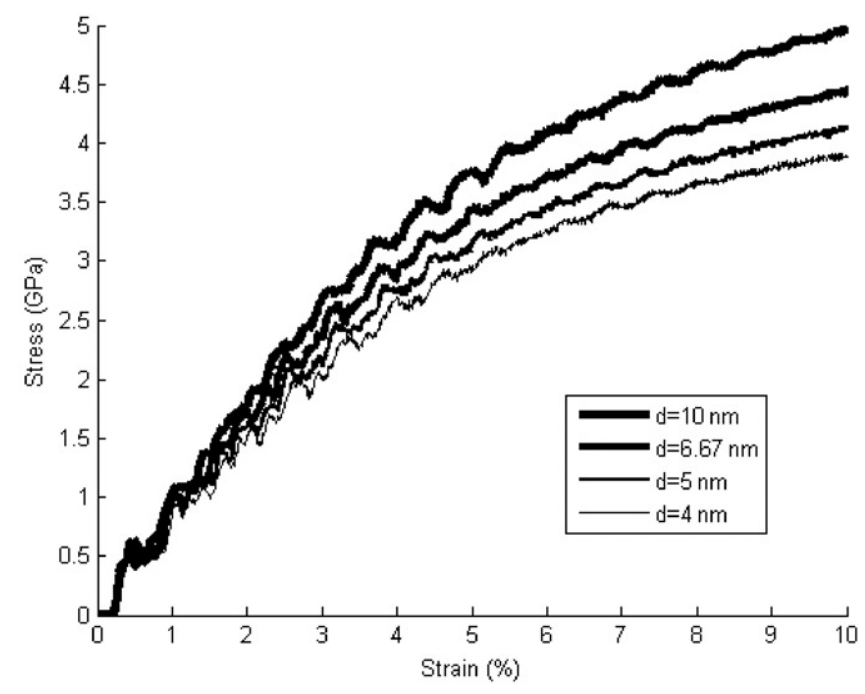

Figure 6. Grain-size dependence of the stress-strain response of nc copper predicted by the model at extreme loading rate of $5 \times 10^{8} \mathrm{~s}^{-1}[14]$.

oscillations are observed in the stress-strain curves. This makes it difficult to identify a well-defined yield stress. For comparison purposes, we conventionally extract points on the stress-strain curves that would elastically unload to $2 \%$ permanent deformation. Similar approaches for identifying yield points in nanocrystalline metals have been adopted in $[44,45]$. The extracted conventional values of 'yield' stress are plotted on a Hall-Petch plot $\left(\sigma_{y}\right.$ vs. $\left.d^{-1 / 2}\right)$ and compared with the atomistic results in figure 8 .

It is clear from this figure that, due to the increased presence of grain boundaries, the yield stress decreases with the grain size. In addition, the dependence of the yield stress on the grain size follows a reverse Hall-Petch relation with a similar slope $k$ obtained by atomistic simulation.

A similar approach has been followed for comparing model predictions of the size dependency of the yield stress under quasi-static loading with the experimental results in $[33,34]$. In this case, the sample size was $100 \mathrm{~nm}$ and three different simulations with grain sizes $d=20 \mathrm{~nm}, d=25 \mathrm{~nm}$ and $d=33.3 \mathrm{~nm}$, corresponding to designations $1 \times 5 \times 5,1 \times 4 \times 4$ and $1 \times 3 \times 3$ respectively, were conducted. The same grain-boundary thickness $h=1 \mathrm{~nm}$ as in the high strain-rate simulations was adopted. The resulting stress-strain curves are shown in figure 7 . It can be observed in this figure that the same softening tendency with the reduction of the grain size is obtained. Under quasi-static conditions, the stress-strain curves do not exhibit oscillations and a conventional yield stress at a $0.2 \%$ offset permanent strain may be defined. The extracted yield stress values as well as the experimental results reproduced from [33, 34] were added to the Hall-Petch plot in figure 8, showing a reasonable agreement between simulations and experiments. This suggests that the vast discrepancy in the yield stress of nc metals between experiments and atomistic simulations may be at least partially 


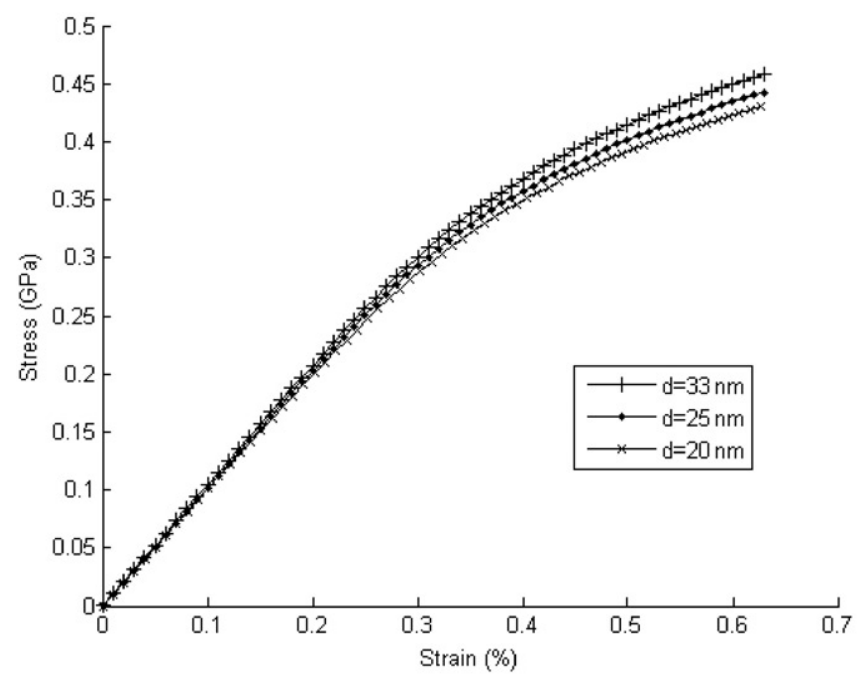

Figure 7. Grain-size dependence of the stress-strain response of nc copper predicted by the model under quasi-static loading [33].

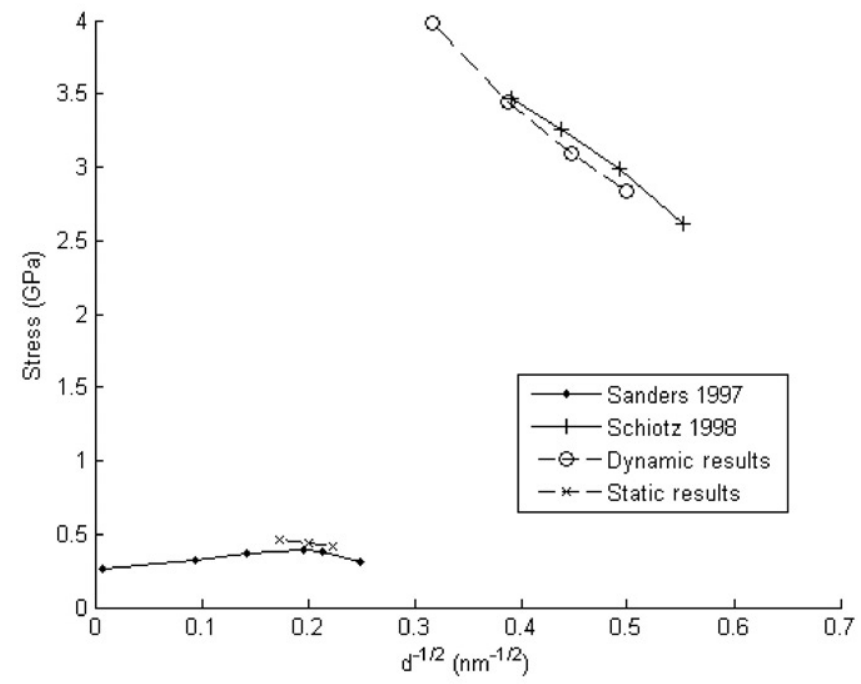

Figure 8. Summary of the grain-size dependence of the yield stress in nc copper. The plot compares the predictions of the continuum model proposed with atomistic results at extreme loading rates [14] and experimental quasi-static results [34].

explained by rate-dependency effects. However, it should also be emphasized that the proposed model overplays the role of rate-dependency, as other potentially important sources of discrepancy owing to the idealizations of atomistic models are ignored. 


\section{Conclusions}

A continuum model describing grain-boundary deformation mechanisms in nc metals has been presented. The modelling strategy explicitly accounts for the deformation of individual grains and consists of a phenomenological constitutive approach formulated within the framework of variational constitutive updates to describe sliding and opening deformation at the grain boundary.

The model captures the salient features of the effective stress-strain response predicted by atomistic models and is also consistent with experimental observations. These include: the onset of yield and yield response of nanocrystals attributable to grain-boundary deformation, the decrease in the yield stress for decreasing grain sizes at the nanoscale which follows an inverse Hall-Petch relation and the rate-dependence of the yield stress.

One of the advantages of the continuum modelling approach is that it enables the analysis of large nc samples and complex boundary conditions, as the need to account for the individual atoms is avoided. It is also important to highlight that the continuum modelling approach does not suffer from the time step limitations of MD and, thus, enables the consideration of a vast range of loading rates including static loads.

\section{Acknowledgments}

The support of the US Department of Energy through the ASC Center for the Simulation of the Dynamic Response of Materials (DOE W-7405-ENG-48, B523297) is gratefully acknowledged.

\section{References}

[1] K.S. Kumar, H. Van Swygenhoven and S. Suresh, Acta Mater. 515743 (2003).

[2] C.J. Youngdahl, P.G. Sanders, J.A. Eastman, et al. Scripta Mater. 37809 (1997).

[3] S.R. Agnew, B.R. Elliott, C.J. Youngdahl, et al. Mater. Sci. Eng. A285 391 (2000).

[4] H.V. Swygenhoven, Science 29666 (2002).

[5] H.V. Swygenhoven and J.R. Weertman, Scripta Mater. 49625 (2003).

[6] K.W. Jacobsen and J. Schiøtz, Nature Mater. 115 (2002).

[7] E.O. Hall, Proc. Phys. Soc. Lond. B64 747 (1951).

[8] N.J. Petch, J. Iron Steel Inst. 17425 (1953).

[9] A.H. Chokshi, A. Rosen, J. Karch, et al. Scripta Metalrg. 231679 (1989).

[10] H.W. Song, S.R. Guo and Z.Q. Hu, Nanostruct. Mater. 11203 (1999).

[11] M.A. Haque and M.T.A. Saif, Scripta Mater. 47863 (2002).

[12] C.A. Schuh, T.G. Nieh and H. Iwasaki, Acta Mater. 51431 (2003).

[13] M.A. Meyers, A. Mishra and D.J. Benson, Prog. in Mater. Sci. 51427 (2006).

[14] J. Schiøtz, F.D. Di Tolla and K.W. Jacobsen, Nature 391561 (1998).

[15] S. Yip, Nature 391532 (1998).

[16] H. Van Swygenhoven, M. Spaczer, A. Caro, et al. Phys. Rev. B: Condens. Matter 6022 (1999).

[17] J. Schiøtz, T. Vegge, F.D. Di Tolla, et al. Phys. Rev. B 6011971 (1999).

[18] V. Yamakov, D. Wolf and S.R. Phillpot, Nature Mater. 11 (2002).

[19] J. Schiøtz and K.W. Jacobsen, Science 3011357 (2003).

[20] H.S. Kim, Y. Estrin and M.B. Bush, Acta Mater. 48493 (2000). 
[21] B. Jiang and G.J. Weng, Metallurg. Mater. Trans. A 34A 765 (2003).

[22] H.H. Fu, D.J. Benson and M.A. Meyers, Acta Mater. 492567 (2001).

[23] R. Schwaiger, B. Moser, M. Dao, et al. Acta Mater. 515159 (2003).

[24] H.-H. Fu, D.J. Benson and M.A. Meyers, Acta Mater. 524413 (2004).

[25] Y.J. Wei, C. Su and L. Anand, Acta Mater. 543177 (2006).

[26] Y.J. Wei and L. Anand, J. Mech. Phy. Solids 522587 (2004).

[27] D.H. Warner, F. Sansoz and J.F. Molinari, Int. J. Plast. 22754 (2006).

[28] M. Ortiz and A. Pandolfi, Int. J. Numer. Meth. Eng. 441267 (1999).

[29] R. Radovitzky and M. Ortiz, Comp. Meth. Appl. Mech. Eng. 172203 (1999).

[30] M. Ortiz and L. Stainier, Comp. Meth. Appl. Mech. Eng. 171419 (1999).

[31] A.C. Lund and C.A. Schuh, Acta Mater. 533193 (2005).

[32] E.M. Bringa, A. Caro, Y.M. Wang, et al. Science 3091838 (2005).

[33] P.G. Sanders, J.A. Eastman and J.R. Weertman, Acta Metallurg. 454019 (1997).

[34] P.G. Sanders, C.J. Youngdhal and J.R. Weertman, Mater. Sci. Eng. A 234-236 77 (1997).

[35] J.E. Marsden and T.J.R. Hughes, Mathematical Foundations Elasticity (Prentice-Hall, Englewood Cliffs, NJ, 1983).

[36] M. Ortiz and A. Pandolfi, Comp. Meth. App. Mechan. Eng. 1932645 (2004).

[37] K. Weinberg, A. Mota and M. Ortiz, Comput. Mech. 37142 (2006).

[38] E. Fancello, J.-Ph. Ponthot and L. Stainier, Int. J. Numer. Meth. Eng. 651831 (2006).

[39] Q. Yang, L. Stainier and M. Ortiz, J. Mechan. Phys. Solids 54401 (2006).

[40] L.J. Lubliner, Int. J. Non-Lin. Mech. 7237 (1972).

[41] L.J. Lubliner, Acta Mechan. 17109 (1973).

[42] P. Heino and E. Ristolainen, Phil. Mag. A 81957 (2001).

[43] S. Takeuchi, Scripta Mater. 441483 (2001).

[44] L. Lu, S.X. Li and K. Lu, Scripta Mater. 451163 (2001).

[45] G.J. Fan, H. Choo, P.K. Liaw, et al. Acta Mater. 541759 (2006). 